\title{
GCU
}

Glasgow Caledonian

University

University for the Common Good

\section{The effect of ocular rigidity upon the characteristics of saccadic eye movements}

Alhazmi, Mohammed; Seidel, Dirk; Gray, Lyle S.

Published in:

Investigative Ophthalmology and Visual Science

DOI:

10.1167/iovs.13-13688

Publication date:

2014

Document Version

Publisher's PDF, also known as Version of record

Link to publication in ResearchOnline

Citation for published version (Harvard):

Alhazmi, M, Seidel, D \& Gray, LS 2014, 'The effect of ocular rigidity upon the characteristics of saccadic eye movements', Investigative Ophthalmology and Visual Science, vol. 55, no. 3, pp. 1251-1258.

https://doi.org/10.1167/iovs.13-13688

\section{General rights}

Copyright and moral rights for the publications made accessible in the public portal are retained by the authors and/or other copyright owners and it is a condition of accessing publications that users recognise and abide by the legal requirements associated with these rights.

Take down policy

If you believe that this document breaches copyright please view our takedown policy at https://edshare.gcu.ac.uk/id/eprint/5179 for details of how to contact us. 


\title{
The Effect of Ocular Rigidity Upon the Characteristics of Saccadic Eye Movements
}

\author{
Mohammed Alhazmi, ${ }^{1,2}$ Dirk Seidel, ${ }^{1}$ and Lyle S. Gray ${ }^{1}$ \\ ${ }^{1}$ Department of Life Sciences, Glasgow Caledonian University, Glasgow, United Kingdom \\ ${ }^{2}$ Department of Optometry and Vision Sciences, King Saud University, Riyadh, Saudi Arabia
}

Correspondence: Mohammed Alhazmi, 70 Cowcaddens Road, Glasgow, Lanarkshire G4 0BA, UK;

Mohammed.Alhazmi@gcu.ac.uk.

Submitted: December 6, 2013

Accepted: January 21, 2014

Citation: Alhazmi M, Seidel D, Gray LS. The effect of ocular rigidity upon the characteristics of saccadic eye movements. Invest Ophthalmol Vis Sci. 2014;55:1251-1258. DOI:10.1167/ iovs.13-13688
Purpose. To determine whether variation in ocular rigidity (a quantity that describes the elastic properties of the globe) affects the characteristics of horizontal saccadic eye movements.

MетноDs. Thirty-three young, visually healthy subjects participated with informed consent in the study. Axial length was measured using the IOLMaster ocular biometer. Ocular rigidity coefficients were determined using Schiotz tonometry. Horizontal saccades were stimulated randomly to $40^{\circ}$ in $10^{\circ}$ steps. Eye movements were recorded continuously at a sampling rate of $60 \mathrm{~Hz}$ using the Viewpoint video-eyetracker.

RESults. Peak velocity increased significantly with increasing ocular rigidity $(F[2,263]=$ $30.635, P<0.001)$. Time to peak velocity $(F[2,263]=27.723, P<0.001)$ and total response time $(F[2,263]=21.133, P<0.001)$ decreased significantly with increasing ocular rigidity. Ocular rigidity was significantly positively correlated with peak velocity $\left(R^{2}=0.67, P<\right.$ $0.001)$, and significantly negatively correlated with time to peak velocity $\left(R^{2}=0.64, P<\right.$ $0.001)$, and total response time $\left(R^{2}=0.62, P<0.001\right)$.

Conclusions. The known relationship of ocular rigidity with myopia can be extended to shorter hyperopic eyes, which are found to have higher ocular rigidity. The dynamic characteristics of saccadic eye movements are found to vary systematically with ocular rigidity. These findings suggest that the structural characteristics of the eye are an important factor in determining dynamic characteristics of eye movements.

Keywords: ocular rigidity, axial length, saccadic eye movements, peak velocity
Saccadic eye movements are an integral part of the $\checkmark$ visuomotor system. ${ }^{1}$ Many studies have described the key characteristics of saccadic eye movements such as peak velocity (PV), time to peak velocity (TPV), total response time (TRT), and latency. Peak velocity varies with stimulus size, increasing from $114^{\circ} / \mathrm{s}$ for a $5^{\circ}$ stimulus $^{2}$ to $445^{\circ} / \mathrm{s}$ for a $20^{\circ}$ stimulus, ${ }^{3-5}$ and to more than $500^{\circ} / \mathrm{s}$ for a $30^{\circ}$ stimulus. ${ }^{4,5}$ Time to peak velocity varies with stimulus size from $16 \mathrm{~ms}$ for a $3^{\circ}$ stimulus to $22.5 \mathrm{~ms}$ for a $9^{\circ}$ stimulus. $^{6}$ Total response time increases with stimulus size from $22 \mathrm{~ms}$ for a $4^{\circ}$ stimulus $^{7}$ to 68 ms for $10^{\circ}$ stimulus and to $91 \mathrm{~ms}$ for a $20^{\circ}$ stimulus. $^{3}$ Latency varies from 136 to $275 \mathrm{~ms}$ in visually healthy observers depending upon eye recording techniques, experimental design, and age. ${ }^{5,8,9}$

Refractive error arises primarily as a result of differences in axial length, with shorter axial lengths producing hyperopia, ${ }^{10,11}$ and longer axial lengths leading to myopia. ${ }^{10,12-14}$ Recent work has shown that the increase in ocular size in myopia is found in all planes (Miller JM, et al. IOVS 2004:ARVO E-Abstract 2388; Logan NS, et al. IOVS 2005: ARVO E-Abstract 4266; and Refs. 12-14), although the enlargement is largest in the axial plane. ${ }^{14,15}$ It is well established that ocular rigidity (OR) is reduced in larger myopic eyes compared with emmetropic ${ }^{16-19}$ and hyperopic eyes. ${ }^{16,17,20}$ The low OR found in myopia is associated with weakening and reduced tensile strength of the sclera ${ }^{19,21,22}$ and is due primarily to a thinning of collagen fiber bundles and a reduction in the size of the individual collagen fibrils. ${ }^{19,22}$

Copyright 2014 The Association for Research in Vision and Ophthalmology, Inc www.iovs.org | ISSN: 1552-5783
Relatively little work has been done to examine whether these variations in OR influence the dynamic characteristics of saccadic eye movements. Robinson ${ }^{23}$ found ocular mass to be negligible in determining saccadic time course as increasing the inertia of the system by $9650 \%$ did not affect the time course of saccadic responses. These findings demonstrate that extraocular muscle force can easily overcome the ocular mass and associated inertia by several orders of magnitude. Robinson ${ }^{23}$ did not measure OR, so was unable to examine the effect of OR upon saccadic eye movement parameters. A recent study of 40 subjects (20 emmetropes and 20 myopes) found that highly myopic eyes ( $>6$ diopters [D]) showed significantly slower mean velocities (up to $20^{\circ}$ /s slower) for $7.5^{\circ}, 15^{\circ}$, and $22.5^{\circ}$ of saccadic eye movement. ${ }^{24}$ In contrast, Hartwig et al. ${ }^{25}$ found that myopic subjects (mean spherical equivalent [MSE] ranged from -0.88 to $-7.13 \mathrm{D}$ ) and emmetropes had similar saccadic eye movement characteristics, although they examined only small $10^{\circ}$ movements.

The purpose of the present study is to determine whether a normal population variation in OR can affect the characteristics of saccadic eye movements in both long (myopic) and short (hyperopic) eyes.

\section{Materials ANd Methods}

\section{Subjects}

Thirty-three visually healthy subjects (19 male and 14 female) participated in the experiment. There were 15 myopic 


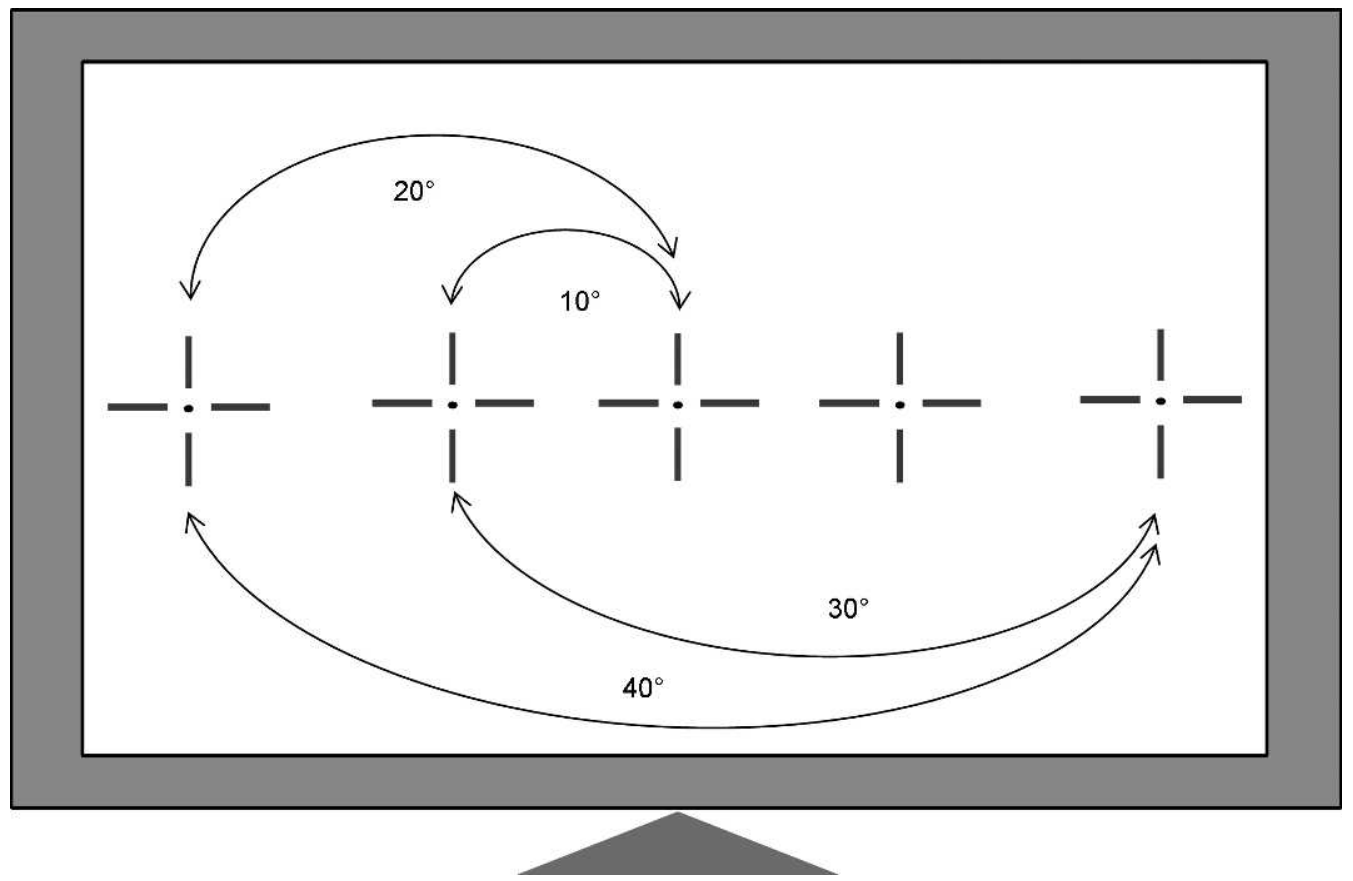

Figure 1. The stimulus was composed of a central black dot $0.6^{\circ}$ in diameter with surrounding fixation bars $0.4^{\circ}$ width, and $3.6^{\circ}$ in length. The fixation bars were placed at $0.8^{\circ}$ from the central fixation dot. Stimulus displacements were calculated to produce $10^{\circ}, 20^{\circ}, 30^{\circ}$, and $40^{\circ}$ saccades.

subjects, 10 emmetropic, and 8 hyperopic, ranging in age from 18 to 36 years (mean $\pm \mathrm{SD}, 23.52 \pm 5.11$ years). Ethical approval was obtained from the School of Life Sciences ethics committee and the study was conducted according to the tenets of the Declaration of Helsinki. Informed consent was obtained from each subject prior to beginning the study. Exclusion criteria were any ocular or systemic pathology, including previous history of ocular surgery, history of trauma, ocular or systemic medication, binocular vision abnormality, and astigmatism greater than $2.00 \mathrm{D}$. Subjects with a range of MSE refractive errors from $-10.00 \mathrm{D}$ to $+6.00 \mathrm{D}$ were recruited to the study.

Visual acuity was fully corrected to 0.0 LogMAR or better with daily disposable soft contact lenses (Focus Dailies, Nelfilcon A [water content 69\%]; CIBA VISION, Duluth, GA).

\section{Measurements of Ocular Parameters}

Refractive error was measured using standard subjective refraction methods. Axial length was determined by averaging three measurements using noncontact partial coherence interferometry (IOLMaster; Carl Zeiss, London, UK). Intraocular pressure (IOP) was measured using a Schiotz tonometer (Biro Ophthalmic Instruments, Burladingen, Germany) with weights of $5.5,7.5$, and $10 \mathrm{~g}$. Mean IOP values, based on three recordings with each weight, were used to derive OR using Friedenwald's Nomogram. ${ }^{26,27}$ Previous work has shown measurement of IOP using Schiotz tonometry has good repeatability. ${ }^{28-30}$

\section{Stimulus}

High contrast (90\%) stimuli (Fig. 1) were presented randomly upon a computer monitor (Mitsubishi cathode ray tube, width $49.5 \mathrm{~cm}$, height $49.3 \mathrm{~cm}$, resolution $640 \times 480$ at $60 \mathrm{~Hz}$ and a brightness of $100 \mathrm{~cd} / \mathrm{m}^{2}$ for a full white background; Mitsubishi Electric Corporation, Nagasaki, Japan) to elicit saccades of varying magnitude from $10^{\circ}$ to $40^{\circ}$ in both rightwards and leftwards directions.

Subjects were aligned centrally to the computer monitor, and the stimuli aligned vertically with the eyes ensuring that only horizontal saccades were elicited. Head movements were restricted by means of a chin and forehead support, with a restraining head strap. The experiment was performed binocularly at a viewing distance of $40 \mathrm{~cm}$.

\section{Stimulus Presentation}

For the $10^{\circ}$ and $20^{\circ}$ saccades, the stimulus was presented randomly at either the primary central position or at $10^{\circ}$ and $20^{\circ}$ and then moved right or left. For the $30^{\circ}$ and $40^{\circ}$ saccades the stimulus was presented at $10^{\circ}$ and $20^{\circ}$ away from the primary position in one direction and then moved in the opposite direction to produce the required stimulus for $30^{\circ}$ or $40^{\circ}$ (Fig. 1). Stimuli were presented randomly for a minimum of 10 seconds at each location.

\section{Eye Movement Recording}

Eye movements were recorded continuously at a sampling rate of $60 \mathrm{~Hz}$ using the Viewpoint infrared video eyetracker (Arrington Research, Scottsdale, AZ). This eyetracker has an optimum resolution of $0.15^{\circ}$ of visual arc as quoted by the manufacturer. A full examination session lasted 45 minutes and the subjects were given regular breaks to avoid fatigue.

Recordings were rejected where blinks occurred during the response and where latency was shorter than $90 \mathrm{~ms}$ or longer than $450 \mathrm{~ms}$ as such responses could be due to anticipation or lack of attention, respectively. ${ }^{31,32}$ Every response was repeated 12 times and a minimum of seven clean recordings were averaged for each.

\section{Data Analysis}

An algorithm written in MS Visual Basic (Microsoft Corporation, Redmond, WA) was used to detect and remove blinks. 


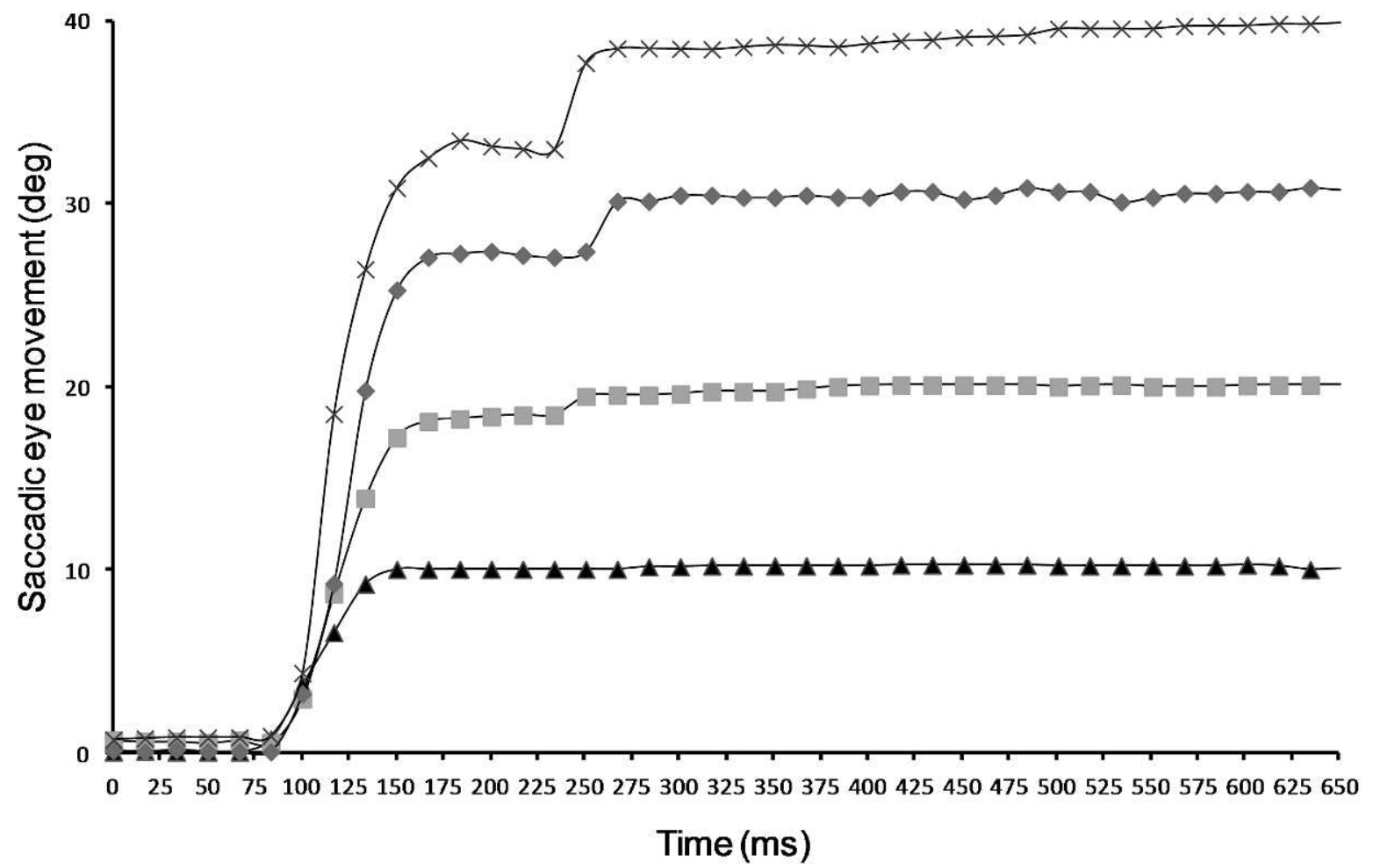

Figure 2. Typical saccadic responses for subject SK for $10^{\circ}, 20^{\circ}, 30^{\circ}$, and $40^{\circ}$ plotted as original eye position against time.

Intersubject variations in interpupillary distance (IPD) were accounted for in the analysis. An example of typical eye movement recordings for one subject is shown in Figure 2.

The following saccadic response characteristics were analyzed offline: PV, TPV, TRT, and latency. Saccadic response onset and completion were determined as the points where eye movement velocity exceeded or fell below $5^{\circ} / \mathrm{s}^{33,34}$ The PV was obtained by differentiation of the eye movement recordings after blink removal. Latency was defined as the time between the target onset and the onset of the saccadic response.

Subjects were divided into three arbitrary groups with equal subject numbers according to their OR as follows:

1. High OR (ranged from $0.0175-0.0188 \mathrm{~mm} \mathrm{Hg} / \mu \mathrm{L}$, mean $\pm \mathrm{SD}=0.0182 \pm 0.0004 \mathrm{~mm} \mathrm{Hg} / \mu \mathrm{L})$ : the axial length for this group ranged from 21.31 to $23.29 \mathrm{~mm}$ mean \pm $\mathrm{SD}=22.73 \pm 0.76 \mathrm{~mm}$;

2. Medium OR (ranged from $0.0148-0.0168 \mathrm{~mm} \mathrm{Hg} / \mu \mathrm{L}$, mean $\pm \mathrm{SD}=0.0157 \pm 0.0006 \mathrm{~mm} \mathrm{Hg} / \mu \mathrm{L})$ : axial length ranged from 23.52 to $24.77 \mathrm{~mm}$, mean $\pm \mathrm{SD}=24.00 \pm$ $0.50 \mathrm{~mm}$; and

3. Low OR (ranged from $0.0132-0.0146 \mathrm{~mm} \mathrm{Hg} / \mu \mathrm{L}$, mean $\pm \mathrm{SD}=0.0142 \pm 0.0009 \mathrm{~mm} \mathrm{Hg} / \mu \mathrm{L})$ : Axial length ranged from 24.82 to $27.76 \mathrm{~mm}$, mean $\pm \mathrm{SD}=25.90 \pm$ $0.93 \mathrm{~mm}$.

All reported data were for the right eye and for centrifugal saccades only. Although the $30^{\circ}$ and $40^{\circ}$ saccades were crossing the primary position, the eye always landed centrifugally upon the completion of these movements.

Univariate general linear ANOVA was performed on PV, TPV, TRT, and latency with the fixed factors of OR, direction of movement (adduction and abduction), and stimulus size. Post hoc comparisons were carried out using the Tukey HSD test.

\section{Results}

\section{Relationship Between OR and Axial Length/ Refractive Error}

Axial length varied from 21.31 to $27.67 \mathrm{~mm}$ (mean $\pm \mathrm{SD}=$ $24.21 \pm 1.53 \mathrm{~mm}$ ). Ocular rigidity and axial length were significantly negatively correlated, $\left(R^{2}=0.84, F[1,32]=\right.$ 159.080, $P<0.001$; Fig. 3). Mean OR for the highest OR group was significantly greater $(F[1,32]=169.214, P<0.001)$ than both the medium OR group $(P<0.001)$ and low OR group $(P$ $<0.001)$, and the medium OR group was significantly greater than the low OR group $(P<0.001)$.

Ocular rigidity and MSE refractive error were significantly positively correlated, $\left(R^{2}=0.76, F[1,32]=99.058, P<0.001\right.$; Fig. 4).

\section{Peak Velocity}

Peak velocity (Fig. 5) increased significantly with increasing OR $(F[2,263]=30.635, P<0.001)$. The high OR group had a significantly faster PV than the medium OR $(P<0.001)$ and the low OR group $(P<0.001)$. The medium OR group showed a significantly faster PV compared with the low OR group $(P=$ 0.016). Peak velocity increased significantly with increasing size of saccadic eye movement $(F[3,263]=711.261, P<$ 0.001 ). Peak velocity for $10^{\circ}$ was significantly slower than $20^{\circ}$, $30^{\circ}$, and $40^{\circ}(P<0.001$ for all comparisons $), 20^{\circ}$ was significantly slower than $30^{\circ}$ and $40^{\circ}(P<0.001$ for all comparisons) and $30^{\circ}$ was significantly slower than $40^{\circ}(P<$ 0.001). Peak velocity was significantly faster for abductive movements $(F[1,263]=7.037, P=0.044)$. There was an interaction between the factors of stimulus size and OR as the difference in PV between the OR groups increases significantly with increasing stimulus size $(F[6,263]=2.787, P=0.012)$. 


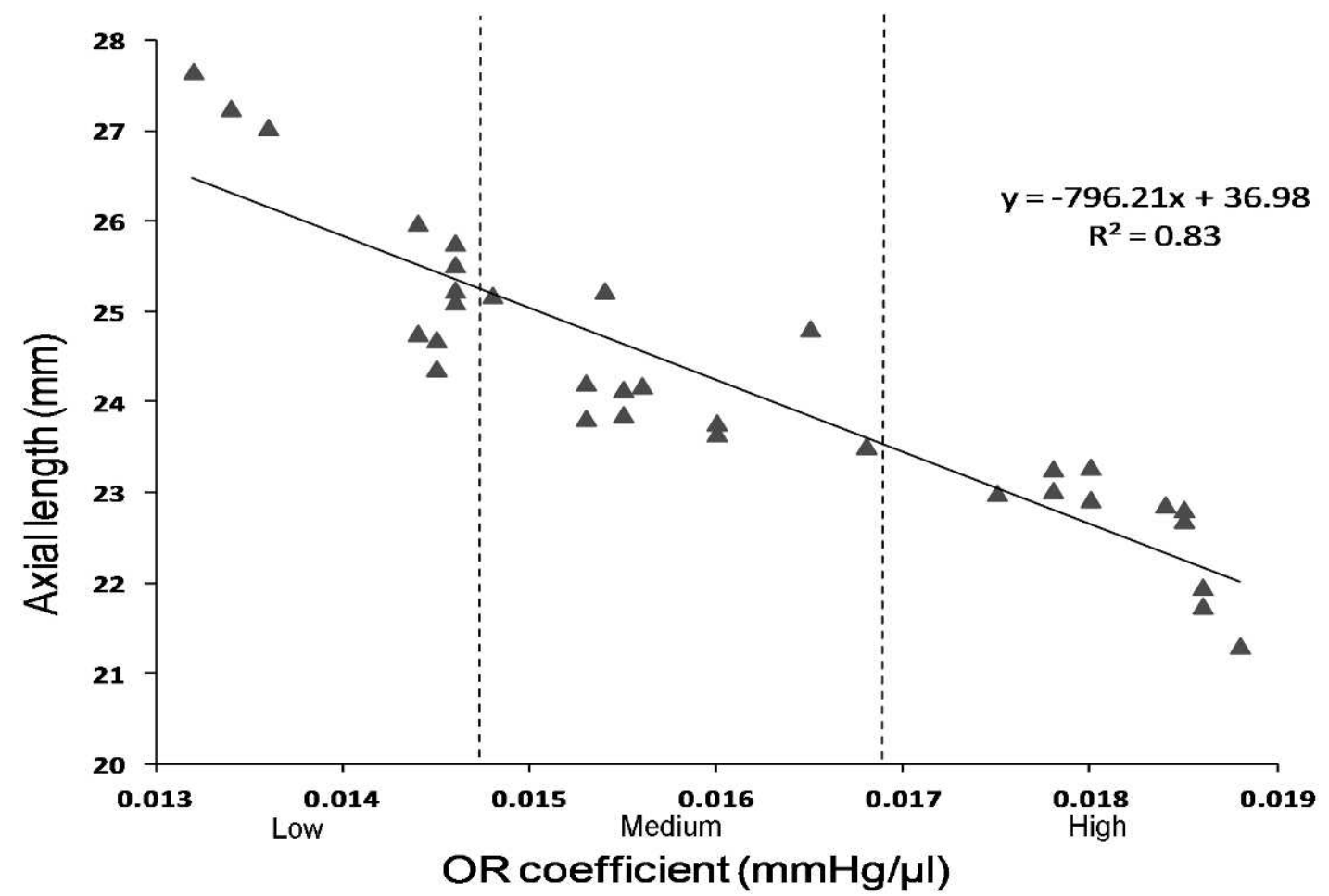

Figure 3. The relationship between the axial length and OR

The asymmetry between abduction and adduction did not vary significantly between the OR groups.

\section{Time to Peak Velocity}

Time to peak velocity (Fig. 6) varied significantly between OR groups $(F[2,263]=27.723, P<0.001)$. The high OR group had a significantly shorter TPV than the medium OR $(P<$ $0.001)$ and the low OR group $(P<0.001)$. The medium OR group showed a significantly shorter TPV compared with the low OR group $(P=0.004)$. Time to peak velocity was also found to increase significantly with increasing stimulus size ( $F$ $[3,263]=176.974, P<0.001)$. Time to peak velocity was

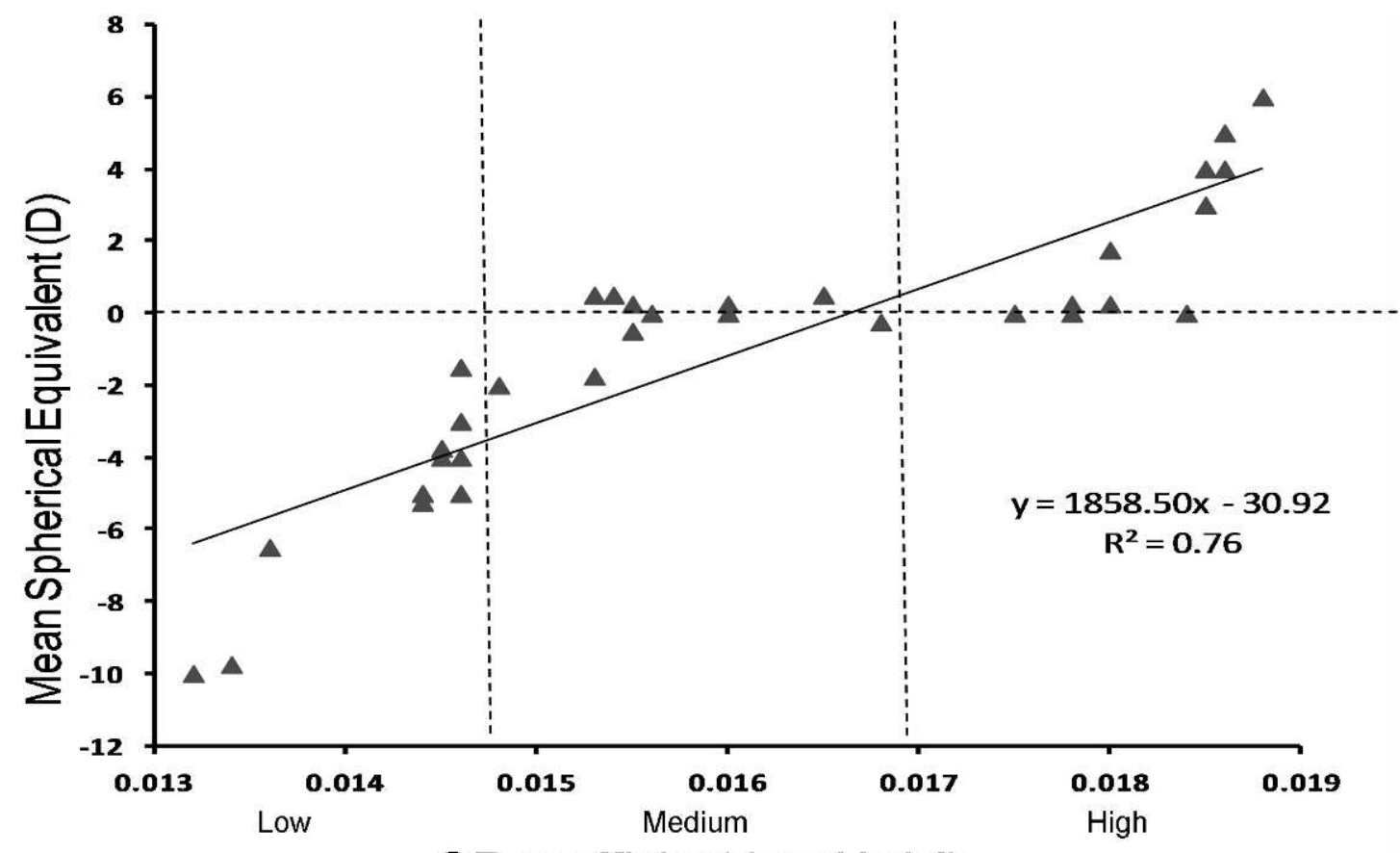

Figure 4. The relationship between the MSE and OR 


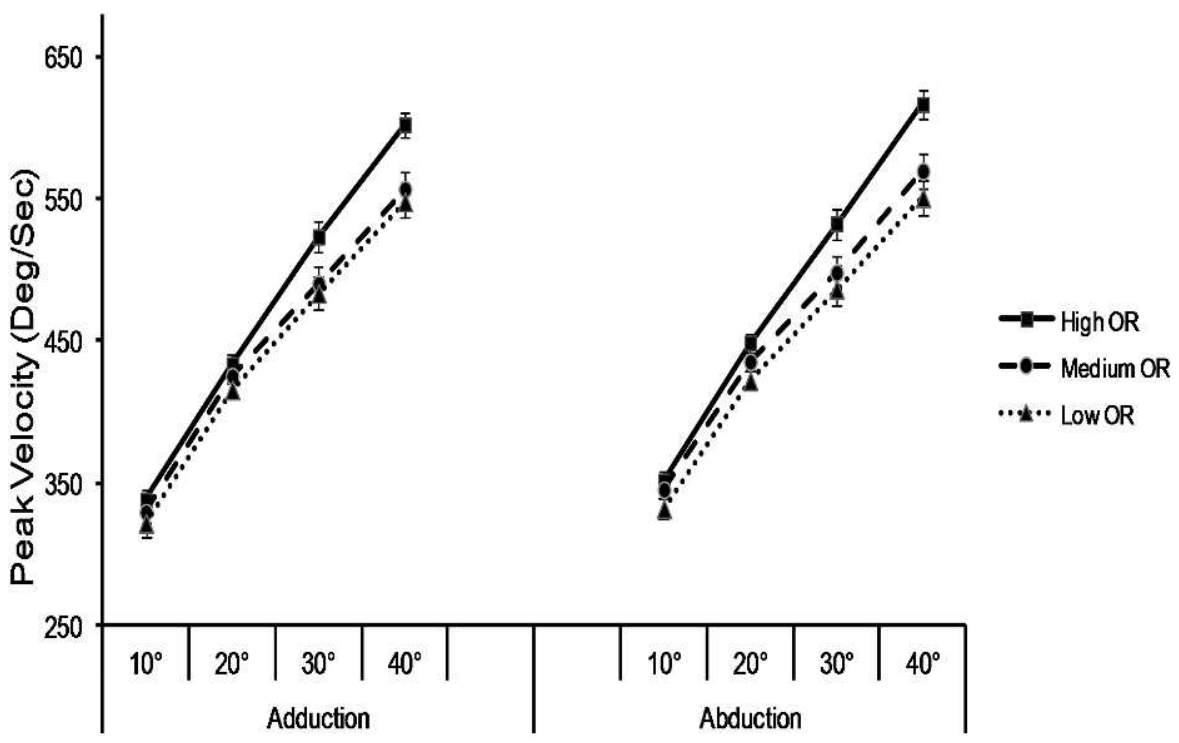

Figure 5. Peak velocity for all stimulus sizes in the three subject groups. For clarity, error bars represent the SEM.

significantly shorter for abductive eye movements $(F[1,263]=$ 13.004, $P<0.001)$. There was an interaction between the factors of stimulus size and OR where the difference in TPV between the OR groups increases as the stimulus size increases $(F[6,263]=3.190, P=0.005)$. The asymmetry between abduction and adduction was not significantly different between the OR groups.

\section{Total Response Time}

Total response time (Fig. 7) varied significantly between the OR groups $(F[2,263]=21.133, P<0.001)$. The high OR group had a significantly shorter TRT than the medium OR $(P<$ $0.001)$ and the low OR group $(P<0.001)$. The medium OR group showed a significantly shorter TRT compared with the low OR group $(P=0.006)$. Total response time was found to be greater with increasing stimulus size $(F[3,263]=71.553, P<$ 0.001). There was an interaction between the factors of groups increases significantly with increasing the stimulus size (F $[6,263]=3.368, P=0.003)$. The asymmetry between abduction and adduction was not significantly different between the OR groups.

\section{Latency}

No significant variation in latency was found between the OR groups. There was a significant variation in latency with stimulus size $(F[3,263]=10.522, P<0.001)$ with the $10^{\circ}$ response having significantly shorter latency than $20^{\circ}(P<$ $0.001), 30^{\circ}(P=0.001)$, and $40^{\circ}(P<0.001)$ responses. No significant difference in latency was found between abductive and adductive eye movements.

\section{Correlation Between OR and PV, TPV, and TRT}

Ocular rigidity was linearly correlated with PV, TPV, and TRT for $40^{\circ}$ saccadic responses. Ocular rigidity was positively

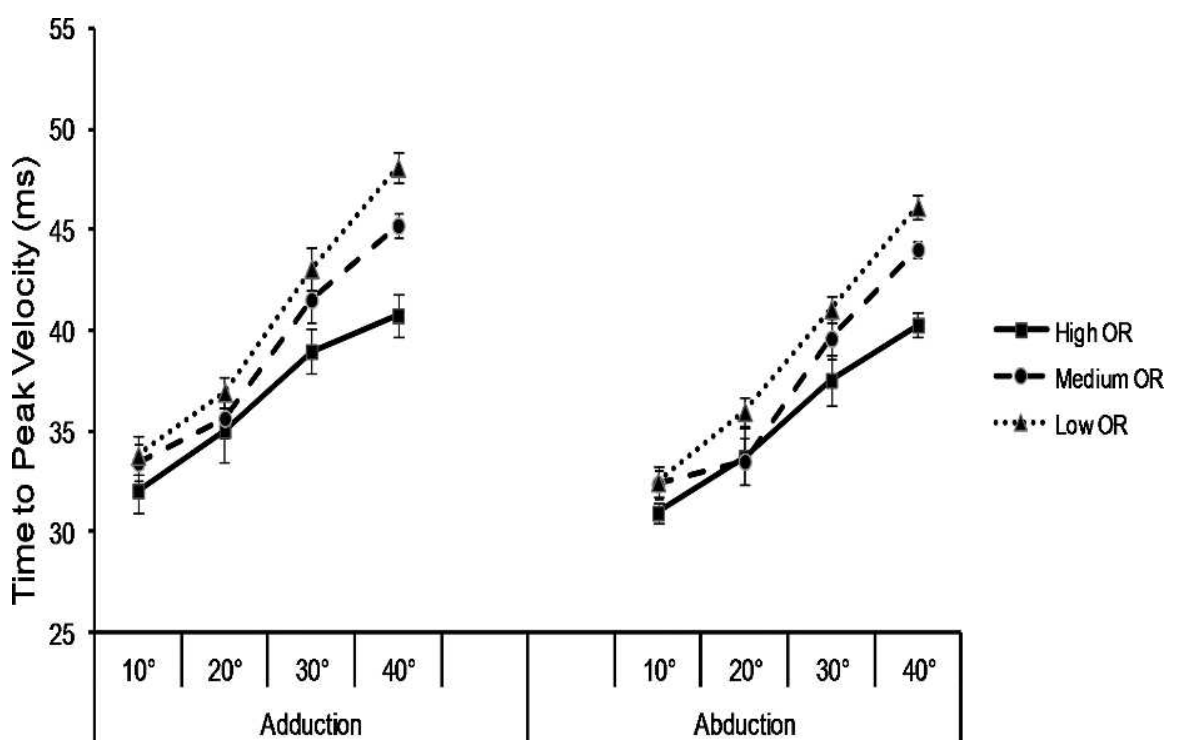

FIGURE 6. Time to peak velocity for all stimulus sizes in the three subject groups. For clarity, error bars represent the SEM. 


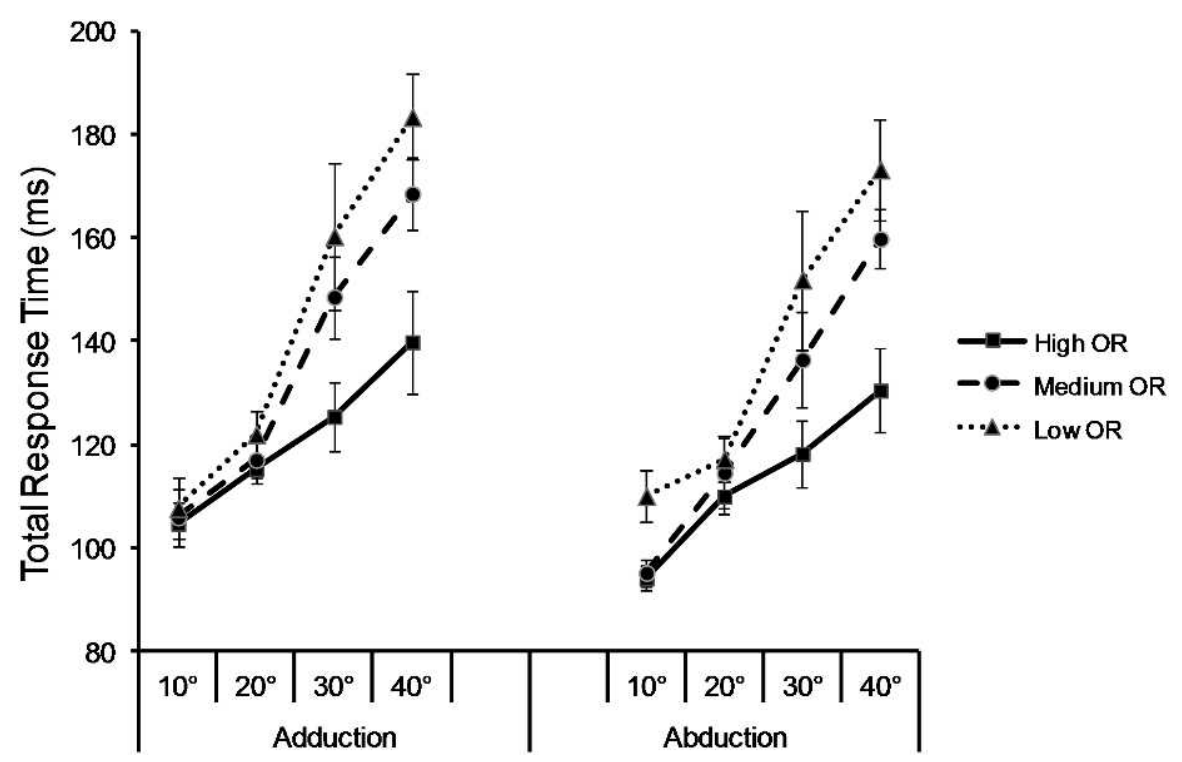

FIGURE 7. Total response time for all stimulus sizes in the three subject groups. For clarity, error bars represent the SEM.

correlated with PV for both abduction $\left(R^{2}=0.68, F=64.55, P\right.$ $<0.001, y=21565 x+233.12)$ and adduction $\left(R^{2}=0.67, F=\right.$ 62.49, $P<0.001, y=19744 x+252.36$ ) such that eyes with high OR reach higher PV during a saccadic eye movement. Additionally, OR was negatively correlated with TPV for both abduction $\left(R^{2}=0.64, F=54.22, P<0.001, y=-1387.9 x+\right.$ 65.768) and adduction $\left(R^{2}=0.70, F=72.89, P<0.001, y=\right.$ $-1967.4 x+76.274)$. Similarly, OR was negatively correlated with TRT for both abduction $\left(R^{2}=0.62, F=50.25, P<0.001\right.$, $y=-14117 x+381.1)$ and adduction $\left(R^{2}=0.57, F=37.60, P<\right.$ $0.001, y=-12970 x+372.14)$.

These correlations between OR and PV and TPV and TRT for $40^{\circ}$ saccadic responses retained significance when only the 10 emmetropic subjects with similar axial lengths (ranged from 23.00-24.19 mm), but with different OR values (ranged from $0.0153-0.018 \mathrm{~mm} \mathrm{Hg} / \mu \mathrm{L}$ ), were examined. Ocular rigidity was positively correlated with $\mathrm{PV}$ for both abduction $\left(R^{2}=0.43, F=\right.$ 6.01, $P=0.039, y=17161 x+299.92)$ and adduction $\left(R^{2}=\right.$ $0.48, F=7.36, P=0.027, y=12212 x+373.71)$. Ocular rigidity was negatively correlated with TPV for both abduction $\left(R^{2}=\right.$ $0.49, F=7.92, P=0.023, y=-1201.4 x+63.054)$ and adduction $\left(R^{2}=0.49, F=7.97, P=0.022, y=-1569.9 x+\right.$ 70.576). Similarly, OR was negatively correlated with TRT for both abduction $\left(R^{2}=0.55, F=9.76, P=0.014, y=-3874.7 x+\right.$ 220.16) and adduction $\left(R^{2}=0.50, F=7.98, P=0.022, y=\right.$ $-16936 x+446.99)$.

\section{Discussion}

Our study and that of Müller et al. ${ }^{26}$ show that dynamic characteristics of saccadic eye movements are affected by OR. In addition, the findings of this study show that subjects with high OR generate saccadic eye movements with significantly faster PV and significantly shorter TPV than either the medium or low OR groups. We also find that the group with low OR had lower PV than the medium OR group confirming the findings of a previous study, ${ }^{24}$ and we have now extended this finding to subjects with high OR (hyperopes).

While Hartwig et al. ${ }^{25}$ reported that myopes and emmetropes have similar saccadic eye movement characteristics, our findings show that myopes with larger axial length and lower OR have significantly slower saccades compared with both emmetropes and hyperopes with higher values of OR. Hartwig et al..$^{25}$ measured $10^{\circ}$ and $20^{\circ}$ saccades only, whereas in the present study we have measured saccades up to $40^{\circ}$ in both horizontal directions. Our data show that the difference in saccadic characteristics becomes more noticeable for larger saccades (i.e., $30^{\circ}$ and $40^{\circ}$ ). Additionally, Hartwig et al. ${ }^{25}$ used predictable stimuli that might alter the saccadic response characteristics. By comparison, predictability is minimized in our study by randomization of stimulus presentation and by rejecting any response with latency shorter than $90 \mathrm{~ms}$ as a possible anticipatory response. ${ }^{31,32,35}$ Also, only a few of the myopic subjects in the study of Hartwig et al. ${ }^{25}$ had MSE less than or equal to $-4.00 \mathrm{D}$ and our data (Fig. 4) show that the OR of myopic subjects with MSE up to $-3.00 \mathrm{D}$ shows considerable overlap with OR in emmetropic subjects. As Hartwig et al. ${ }^{25}$ did not measure OR, we cannot determine whether there was a difference in OR between their subject groups. The findings of the current study suggest that the range of refractive error examined by Hartwig et al. ${ }^{25}$ means that the OR values in the myopic subjects could be similar to those in the emmetropic subjects and this could be the reason why they fail to find any difference in the characteristics of saccadic eye movements between the two groups. In addition, as we show in the current study, the effect of OR upon the saccadic eye movement characteristics increases with the increasing size of the saccade; hence, it is also possible that the smaller magnitude of saccadic eye movements measured by Hartwig et al. ${ }^{25}$ could be the reason why they do not find any differences in saccadic characteristics between myopic and emmetropic subjects.

Axial length showed the expected negative linear relationship with OR in agreement with previous studies. ${ }^{16-20}$ The longer axial length eyes in the present study have a significantly lower rigidity compared with shorter axial length eyes (Fig. 4). Previous work shows that an increase in axial length is associated with a decrease in scleral thickness ${ }^{19,21,22}$ due primarily to a reduction in the scleral collagen contents resulting from thinning of collagen fiber bundles and a reduction in the size of the individual collagen fibrils. ${ }^{19,22}$ Clearly, this thinning of the sclera has a significant effect upon the OR of the eye. Relatively few studies have measured OR in shorter (hyperopic) eyes ${ }^{16,17,20}$ although Dastiridou et al. ${ }^{20}$ reported recently a linear relationship between OR and axial length in hyperopic subjects, with OR increasing as axial 
length decreased. They showed that this relationship was a continuation of the previously reported relationship between OR and axial length in larger (myopic) eyes. Our findings support those of Dastiridou et al. ${ }^{20}$ as we also find a linear relationship between OR and axial length extending continuously from myopia to hyperopia.

Robinson $^{23}$ demonstrated that ocular mass is negligible in determining the time course of saccadic eye movements. He showed that the force generated by the extraocular muscles was found to be sufficient to overcome an applied weight of 18 $\mathrm{g},{ }^{23}$ which is substantially greater than the typical weight of the eye. Thus, it is unlikely that differences in ocular mass between the subjects would explain the differences in eye movement dynamics identified in the current study or that by Müller et al. $^{24}$

Ocular rigidity is a property of the eye, which could affect the way that the force generated by the extraocular muscles is transferred to the globe. It is possible that in subjects with high OR, and therefore "stiffer" eyes the transfer of force may be more efficient leading to a shorter TPV and a higher PV. The opposite would be true in subjects with low OR. The fact that OR shows a significant relationship with all the saccadic parameters, even in a subgroup of subjects with similar axial lengths suggests that the difference in saccadic characteristics is not related to the mass or inertia of the eye but to the tissue characteristics quantified by OR. A previous study has shown that the thinning of scleral tissue found in larger eyes may be less efficient at transferring the force of the extraocular muscles. ${ }^{36}$ There is no literature reporting eye movement characteristics in subjects with high OR (and shorter eyes) and our findings show the previously described effect of low OR upon saccadic eye movement dynamics ${ }^{24,36}$ can be extended to subjects with high OR (and shorter axial lengths) who are generally hyperopic.

Saccadic eye movement parameters, including PV, TPV, and TRT showed significant response asymmetry between abductive and adductive movement in the present study with the temporal saccades being faster than the nasal saccades in all subject groups. Furthermore, these asymmetries did not differ significantly between the three subject groups. Asymmetries between abduction and adduction have been reported in previous studies,, $37-39$ and have been attributed to the fact that the medial rectus muscle is thicker ${ }^{40}$ and stiffer than the lateral rectus muscle. ${ }^{41,42}$ Additionally, the resistance force exerted by the vertical and the oblique recti during horizontal movement is greater for adductive movements ${ }^{43}$ and the tissue stiffness restraining movement of the globe in the nasal direction is $11 \%$ greater than in the temporal direction. ${ }^{42}$ It has also been shown that arrival of premotor signals at medial rectus motoneurons is delayed compared with that for lateral rectus motoneurons, which may lead to a difference in timing for the activation of the medial and lateral rectus muscles causing asymmetrical saccades. ${ }^{44}$ The data of the present study cannot differentiate between possible causes of asymmetrical saccades; however, it is interesting that our data shows that these saccadic asymmetries are the same in each OR group suggesting that the characteristics of the extraocular muscles do not differ between the groups.

Response latency was the same in all groups irrespective of the size or direction of the stimulus. This suggests that the differences in saccadic eye movement dynamics found in the present study are unrelated to the sensory encoding of the saccadic response, but are more likely to be found in the characteristics of the physical plant, comprising the extraocular muscles and ocular globe. Response latency in this study was longer than that found in other studies, ,5,8,9,31 mainly because the subjects in the current study were naive and had no training prior to participating in the experiment. Also randomization $^{32,45}$ of stimulus presentation would minimize any learning effect, thus reducing anticipation of stimulus movement.

\section{Conclusions}

The known relationship of OR with myopia can be extended to shorter hyperopic eyes, which are found to have higher OR. The dynamic characteristics of saccadic eye movements are found to vary systematically with OR. These findings suggest that the structural characteristics of the eye are an important factor in determining dynamic characteristics of eye movements.

\section{Acknowledgments}

The authors thank the students in the Department of Life Sciences at Glasgow Caledonian University for their participation in this study.

Disclosure: M. Alhazmi, None; D. Seidel, None; L.S. Gray, None

\section{References}

1. Leigh RJ, Zee DS. The Neurology of Eye Movements. New York, NY: Oxford University Press; 2006.

2. Boghen B, Troost BT, Daroff RD, Dell'osso LF, Birkett JE. Velocity characteristics of normal human saccades. Invest Ophthalmol Vis Sci. 1974;13:619-623.

3. Flipse J, Straathof C, Van der Steen J, et al. Binocular saccadic eye movements in multiple sclerosis. J Neurol Sci. 1997;148: 53-65.

4. Hess CW, Muri R, Meienberg O. Recording of horizontal saccadic eye movements methodological comparison between electro-oculography and infrared reflection oculography. Neuro Ophthalmol. 1986;6:189-199.

5. Wilson SJ, Glue P, Ball D, Nutt DJ. Saccadic eye movement parameters in normal subjects. Electroencephalogr Clin Neurophysiol. 1992;86:69-74.

6. Abrams RA, Meyer DE, Kornblum S. Speed and accuracy of saccadic eye movements: characteristics of impulse variability in the oculomotor system. J Exp Psychol Hum Percept Perform. 1989;15:529-543.

7. Rufa A, Mariottini GL, Prattichizzo D, Alessandrini D, Vicino A, Federico A. Video-based eye tracking: our experience with advanced stimuli design for eye tracking software. Ann $N Y$ Acad Sci. 2005;1039:575-579.

8. Abel LA, Troost BT, Dell'osso LF. The effects of age on normal saccadic characteristics and their variability. Vision Res. 1983; 23:33-37.

9. Irving EL, Steinbach MJ, Lillakas L, Babu RJ, Hutchings N. Horizontal saccade dynamics across the human life span. Invest Ophthalmol Vis Sci. 2006;47:2478-2484.

10. Llorente L, Barbero S, Cano D, Dorronsoro C, Marcos S. Myopic versus hyperopic eyes: axial length, corneal shape and optical aberrations. J Vis. 2004;4:288-298.

11. Strang NC, Schmid KL, Carney LG. Hyperopia is predominantly axial in nature. Curr Eye Res. 1998;17:380-383.

12. Greene PR. Mechanical considerations in myopia: relative effects of accommodation, convergence, intraocular pressure, and the extraocular muscles. Am J Optom Physiol Opt. 1980; 57:902-914.

13. Stone RA, Flitcroft DI. Ocular shape and myopia. Ann Acad Med Singapore. 2004;33:7-15.

14. Atchison DA, Jones CE, Schmid KL, et al. Eye shape in emmetropia and myopia. Invest Ophthalmol Vis Sci. 2004;45: 3380-3386. 
15. Deller JFP, O'Connor AD, Sorsby A. X-ray measurement of the diameters of the living eye. Philos Trans $R$ Soc Lond B Biol Sci. 1947;134:456-467.

16. Berisha F, Findl O, Lasta M, Kiss B, Schmetterer L. A study comparing ocular pressure pulse and ocular fundus pulse in dependence of axial eye length and ocular volume. Acta Ophthalmol. 2010;88:766-772.

17. Greene PR. Closed-form ametropic pressure-volume and ocular rigidity solutions. Am J Optom Physiol Opt. 1985;62: 870-878.

18. Pallikaris IG, Kymionis GD, Ginis HS, Kounis GA, Tsilimbaris MK. Ocular rigidity in living human eyes. Invest Ophthalmol Vis Sci. 2005;46:409-414.

19. Pallikaris IG, Dastiridou AI, Tsilimbaris MK, Karyotakis NG, Ginis HS. Ocular rigidity. Expert Rev Ophthalmol. 2010;5: 343-351.

20. Dastiridou AI, Ginis H, Tsilimbaris M, et al. Ocular rigidity, ocular pulse amplitude, and pulsatile ocular blood flow: the effect of axial length. Invest Ophthalmol Vis Sci. 2013;54: 2087-2092.

21. Phillips JR, McBrien NA. Form deprivation myopia: elastic properties of sclera. Ophthalmic Physiol Opt. 1995;15:357362.

22. Summers Rada JA, Sheltona S, Norton TT. The sclera and myopia. Exp Eye Res. 2006;82:185-200.

23. Robinson DA. The mechanics of human saccadic eye movement. J Physiol. 1964;174:245-264.

24. Müller C, Stoll W, Schmäl F. The effect of optical devices and repeated trials on the velocity of saccadic eye movements. Acta Otolaryngol. 2003;123:471-476.

25. Hartwig A, Gowen E, Charman WN, Radhakrishnan H. Binocular saccades in myopes and emmetropes. Optom Vis Sci. 2013;90:980-987.

26. Friedenwald JS. Contribution to the theory and practice of tonometry. Am J Ophthalmol. 1937;20:985-1024.

27. Friedenwald JS. An attempt to remove discrepancies found in the 1954 calibration scale for Schiotz tonometers. Trans Am Acad Ophthalmol Otolaryngol. 1957:108-123.

28. Jackson CR. Schiötz tonometers. An assessment of their usefulness. Br J Ophthalmol. 1965;49:478-484.

29. Anderson DR, Grant WM. Re-evaluation of the Schiotz tonometer calibration. Invest Ophthalmol Vis Sci. 1970;9: 430-446.
30. Chiara GF, Semes LP, Potter JW, Cutter GR, Tucker WR. Portable tonometers: a clinical comparison of applanation and indentation devices. J Am Optom Assoc. 1989;60:105-110.

31. Botzel K, Rottach K, Buttner U. Normal and pathological saccadic dysmetria. Brain. 1993;116:337-353.

32. Ciuffreda K, Tannen B. Eye Movement Basics for the Clinician. St. Louis, MO: Mosby; 1995.

33. Bucci MP, Kapoula Z, Yang Q, Brémond-Gignac D. Latency of saccades, vergence, and combined movements in children with early onset convergent or divergent strabismus. Vision Res. 2006;46:1384-1392.

34. Yang Q, Bucci MP, Kapoula Z. The latency of saccades, vergence, and combined eye movements in children and in adults. Invest Ophthalmol Vis Sci. 2002;43:2939-2949.

35. Hutton SB. Cognitive control of saccadic eye movements. Brain Cogn. 2008;68:327-340.

36. David T, Smye S, James T, Dabbs T. Time-dependent stress and displacement of the eye wall tissue of the human eye. Med Eng Phys. 1997;19:131-139.

37. Collewijn H. Interocular timing differences in the horizontal components of human saccades. Vision Res. 2001;41:34123423 .

38. Kapoula Z, Robins DA. Saccadic undershoot is not inevitable: saccades can be accurate. Vision Res. 1986;26:735-743.

39. Thomas JG. The dynamics of small saccadic eye movements. $J$ Physiol. 1968;200:109-127.

40. Tian S, Nishida Y, Isberg B, Lennerstrand G. MRI measurements of normal extraocular muscles and other orbital structures. Graefes Arch Clin Exp Ophthalmol. 2000;238: 393-404.

41. Al-Hinnawi MF, Barbenel J, Al-Qurainy IA, Dutton GN. Quantitative mechanical assessment of extraocular muscles. Proc Inst Mech Eng. 1991;205:101-108.

42. Collins CC, Carlson MR, Scott AB, Jampolsky A. Extraocular muscle forces in normal human subjects. Invest Ophthalmol Vis Sci. 1981;20:652-664.

43. Robinson DA. A quantitative analysis of extraocular muscle cooperation and squint. Invest Ophthalmol Vis Sci. 1975;14: 801-825.

44. Zee DS, Fitzgibbon EJ, Optican LM. Saccade-vergence interactions in humans. J Neurophysiol. 1992;68:1624-1641.

45. Dickov L, Morrison J. Effects of uncertainty and target displacement on the latency of express saccades in man. Vision Res. 2006;46:2505-2512. 